\title{
Testing for Slope Heterogeneity Bias in Panel Data Models*
}

\author{
Murillo Campello, Antonio F. Galvao, and Ted Juhl
}

\begin{abstract}
Standard econometric methods can overlook individual heterogeneity in empirical work, generating inconsistent parameter estimates in panel data models. We propose the use of methods that allow researchers to easily identify, quantify, and address estimation issues arising from individual slope heterogeneity. We first characterize the bias in the standard fixed effects estimator when the true econometric model allows for heterogeneous slope coefficients. We then introduce a new test to check whether the fixed effects estimation is subject to heterogeneity bias. The procedure tests the population moment conditions required for fixed effects to consistently estimate the relevant parameters in the model. We establish the limiting distribution of the test, and show that it is very simple to implement in practice. Examining firm investment models to showcase our approach, we show that heterogeneity bias-robust methods identify cash flow as a more important driver of investment than previously reported. Our study demonstrates analytically, via simulations, and empirically the importance of carefully accounting for individual specific slope heterogeneity in drawing conclusions about economic behavior.
\end{abstract}

Key Words: Testing, fixed effects, individual heterogeneity, bias, slope heterogeneity, corporate investment

JEL Classification: C12, C23, G31

*The authors are grateful to the editor, associate editor and two anonymous referees for their comments that improved the exposition of the paper. In addition we thank Cecilia Bustamante, Zongwu Cai, George Gao, Erasmo Giambona, Stefan Hoderlein, Hyunseob Kim, Oliver Linton, Mitchell Petersen, Whitney Newey, Suyong Song, Albert Wang, Zhijie Xiao, and the participants at seminars at Boston College, Chapman University, Claremont McKenna College, University of Illinois at Urbana-Champaign, University of Iowa, University of Kansas, University Wisconsin-Milwaukee, 25th Midwest Econometrics Group, and NY Camp Econometrics VIII for their constructive comments and suggestions. Murillo Campello, 369 Sage Hall, Samuel Curtis Johnson Graduate School of Management, Cornell University, Ithaca, NY 14853; (campello@cornell.edu). Antonio Galvao, Department of Economics, University of Arizona, 1130 East Helen Street, McClelland Hall 401, Tucson, AZ 85721; (antoniofgalvao@gmail.com). Ted Juhl, School of Business, Capitol Federal Hall, University of Kansas, Lawrence, KS 66045; (juhl@ku.edu). 


\section{Introduction}

With the increasing availability of large, comprehensive databases, researchers seek to identify and understand richer nuances of microeconomic behavior. Recent economic models introduce behavioral heterogeneity across firms and agents when generating distributions of potential outcomes. To the extent that economic decisions are shaped by these considerations it is natural to consider a methodology that allows for information about the distribution of model inputs (e.g., variances and covariances) to be incorporated in empirical parameter estimates. This paper shows that estimation methods that ignore relations between model input distribution and parameters can lead to biased inferences about microeconomic behavior. Understanding and addressing these problems is key to advancing empiricists' ability to inform theory development.

Empirical work in economics often relies on methods that assume a large degree of homogeneity across individuals. One way to account for some degree of heterogeneity in panel data models is to use the ordinary least squares fixed effects (OLS-FE) estimator. When estimating individual-fixed effects models, one often imposes concomitant assumptions of heterogeneous "intercepts" and homogeneous "slope coefficients" across individuals.

This paper characterizes and addresses the issues of estimation and inference of econometric models in the presence of heterogeneity in individual policy responses ("slope coefficients"). We contribute to the literature by proposing the use of methods that allow researchers to easily identify, quantify, and address estimation issues arising from individual heterogeneity. More generally, our analysis warns researchers about imposing arbitrary homogeneity restrictions when studying complex economic behavior.

We start by describing two main results. First, we show analytically how the presence of individual slope heterogeneity may bias the policy estimates obtained under the OLS-FE framework. We also describe the population moment conditions required for OLS-FE to 
consistently estimate the relevant parameters in the model. Second, we discuss alternative methods that account for slope heterogeneity and produce consistent estimates for the parameters of interest Third, we provide researchers with diagnostic tests to determine whether the use of the OLS-FE approach is warranted in their particular applications.

Importantly, the main contribution of this paper is to develop a new test to identify whether the presence of slope heterogeneity in the data will cause bias in OLS-FE estimates. The OLS-FE is asymptotically biased when the heterogeneous coefficients are correlated with the variation of the regressors. Accordingly, we propose a test for the null hypothesis that there is no correlation between variation of the data and the heterogeneous parameters. Hence, our procedure tests the population moment conditions required for OLS-FE to consistently estimate the relevant parameters in the model. We describe the associated test statistic and derive its limiting distribution. This test is particularly useful for applied researchers in that it follows a standard chi-square distribution, for which critical values are tabulated and widely available.

This paper relates to a literature on testing the hypothesis of slope homogeneity across individuals. There are several proposals in this direction. Pesaran, Smith, and Im (1996) propose an application of the Hausman (1978) test where the OLS-FE estimator is compared with the MG estimator. Phillips and Sul (2003) suggest a "Hausman-type" test in the context of stationary first-order autoregressive panel models, where the cross-section, $N$, is fixed as the time-series, T, goes to infinity. Hsiao (2003) describes a variation of the Breusch and Pagan (1979) test for the slope homogeneity, which is valid when both $N$ and $T$ dimensions tend to infinity. More recently, Pesaran and Yamagata (2008) propose a dispersion type test based on Swamy (1970) type test. Blomquist and Westerlund (2013) propose a test that is robust to general forms of cross-sectional dependence and serial correlation. Compared to the existing procedures, our approach is distinct in several ways. First, differently from this literature where the null hypothesis is slope homogeneity across individuals, the proposed 
methods test the null hypothesis of lack of correlation between variances of the data and the heterogeneous parameters. This is important because it is possible that the individual heterogeneity is such that there is no bias under OLS-FE, and thus existing test procedures would not be able to detect departures from the null hypothesis. Nevertheless, our proposed tests are able to detect such departures since the tests are based on the correlation between variances of the data and the heterogeneous parameters. Second, in the simplest case of the model, the tests do not require the time-series to diverge to infinity.

Monte Carlo simulations assess the finite sample properties of the proposed methods. The experiments suggest that the proposed approaches perform very well in finite samples. The bias of fixed effects estimation can be made arbitrarily large by increasing the magnitude of the covariance between the regression slope and the data variance. Critically, the MG estimator we employ is unaffected by the slope heterogeneity bias. Finally, the new tests possess good finite sample performance and have correct empirical size and power to detect precisely the cases where OLS-FE is biased.

To illustrate the performance of the proposed methods in real-world data, we study empirically the investment model of Fazzari et al. (1988), where a firm's investment is regressed on a proxy for investment demand (Tobin's $Q$ ) and cash flow. Using annual COMPUSTAT data covering a four-decade window, we study slope heterogeneity in investment models contrasting estimates from alternative methodologies. We find that OLS-FE provides biased estimates of the cash flow coefficient. This result is verified by our new test, which strongly rejects the null hypothesis that OLS-FE is consistent. In concrete terms, the estimated coefficient on cash flow increases from 0.057 (0.043) under the OLS (OLS-FE) estimation to 0.291 under the MG estimation; a 6-7-fold increase on the same data. For long panels, the cash flow coefficient is 15 times larger under the MG method. Our results imply that cash flow is a much more relevant driver of investment than previous studies have suggested.

The remainder of the paper is organized as follows. Section 2 describes the biases that 
affect OLS-FE in the presence of individual slope heterogeneity, and discusses the MG estimator. Section 3 presents the proposed test for slope heterogeneity and its generalization. Monte Carlo experiments are discussed in Section 4. In Section 5, we estimate a corporate investment model and compare results from different methods. Section 6 concludes. The proofs for all results are collected in the Supplemental Appendix.

\section{Understanding Heterogeneity Bias}

This section presents the panel data model and characterizes the bias in the OLS-FE estimator when the true econometric model allows for heterogeneous slope coefficients. To guide researchers in their choice of methodology, we also show conditions under which the OLS-FE returns consistent estimates. These conditions are limiting and imply potentially important compromises for researchers' inferences. We also discuss the MG estimator.

\subsection{Biases In The Individual-Fixed Effects Estimator}

When modeling economic behavior, empiricists commonly estimate regression models that allow individuals or firms to have different individual-fixed effects (different "intercepts"), yet impose equal "slope" coefficients across units. As we show next, an incorrect homogeneous slope restriction leads to a bias in the policy estimates one obtains under the ordinary least squares fixed effects (OLS-FE) framework.

Assume the following baseline model for the data generating process

$$
y_{i t}=\alpha_{i}+x_{i t}^{\top} \beta_{i}+u_{i t}, \quad i=1, \ldots, N, t=1, \ldots, T
$$

where $y_{i t}$ is the dependent variable, $x_{i t}$ is a $k \times 1$ vector of exogenous regressors, and $u_{i t}$ is the conditionally mean zero innovation term. The term $\alpha_{i}$ captures individual-specific fixed 
effects, while the slope coefficients $\beta_{i}$ may vary across individuals.

In the presence of slope heterogeneity, one would like to recover the slope coefficient of each individual. In most empirical test settings, however, the objective is to report a summary statistic for policy purposes. In that context, a reasonable quantity to estimate is the average individual slope. As such, the empiricist might try to estimate the parameters $\mathrm{E}\left(\beta_{i}\right)$, the vector representing the average effect from marginal changes in $x_{i t}{ }^{1}$

If all individuals are identical, then the OLS-FE method provides an easy way to estimate the parameters of interest. It is rarely the case, however, that one can a priori justify the assumption of homogeneity in individuals' responses to economic stimuli. Indeed, as discussed above, theoretical modeling and casual observation often suggest otherwise. We now characterize the problems of using the OLS-FE method to estimate the population quantities $\mathrm{E}\left(\beta_{i}\right)=\beta$ (the average individual slope coefficients) in the presence of policy heterogeneity.

The balanced panel model in matrix form is given as

$$
y_{i}=\alpha_{i} \iota_{T}+X_{i} \beta_{i}+u_{i}
$$

for $i=1, \ldots, N$ where $y_{i}$ is a $T \times 1$ vector, $y_{i}=\left(y_{i 1}, \ldots, y_{i T}\right), \iota_{T}$ is a $T \times 1$ vector of ones, $X_{i}$ is a $T \times k$ matrix with rows $x_{i t}^{\top}, X_{i}=\left(x_{i 1}, \ldots, x_{i T}\right)$, and $u_{i}$ is a vector with the errors. The standard fixed effects estimator is calculated based on the implicit assumption that $\beta_{i}$ are the same for each $i$. The formula is given as

$$
\widehat{\beta}_{F E}=\left(\sum_{i=1}^{N} X_{i}^{\top} M_{\iota} X_{i}\right)^{-1} \sum_{i=1}^{N} X_{i}^{\top} M_{\iota} y_{i}
$$

where $M_{\iota}=I_{T}-\iota_{T}\left(\iota_{T}^{\top} \iota_{T}\right)^{-1} \iota_{T}^{\top}, I_{T}$ is an identity matrix of order $T . M_{\iota}$ computes the deviation-from-individual-means.

\footnotetext{
${ }^{1}$ The model in equation (1) can also be interpreted as a random coefficient model. Although we focus on the interpretation of heterogeneous slopes, we refer the reader to Hsiao and Pesaran (2008) and Wooldridge (2010) for detailed discussions of random coefficient models.
} 
It is instructive to rewrite the fixed effects estimator in two different ways. First, if the corresponding inverses exist, we can write

$$
\begin{aligned}
\widehat{\beta}_{F E} & =\sum_{i=1}^{N}\left(\sum_{i=1}^{N} X_{i}^{\top} M_{\iota} X_{i}\right)^{-1}\left(X_{i}^{\top} M_{\iota} X_{i}\right)\left(X_{i}^{\top} M_{\iota} X_{i}\right)^{-1} X_{i}^{\top} M_{\iota} y_{i} \\
& =\sum_{i=1}^{N} W_{i} \widehat{\beta}_{i}
\end{aligned}
$$

where $W_{i}=\left(\sum_{i=1}^{N} X_{i}^{\top} M_{\iota} X_{i}\right)^{-1}\left(X_{i}^{\top} M_{\iota} X_{i}\right)$, and $\widehat{\beta}_{i}=\left(X_{i}^{\top} M_{\iota} X_{i}\right)^{-1} X_{i}^{\top} M_{\iota} y_{i}$. Notice that $\widehat{\beta}_{i}$ is the usual OLS estimator for each $i$.

This representation illustrates this problem more clearly. We wish to estimate $\mathrm{E}\left(\beta_{i}\right)$, the "average" slope. Then,

$$
\widehat{\beta}_{F E}-\mathrm{E}\left(\beta_{i}\right)=A^{-1}(B+C),
$$

where $A=\frac{1}{N} \sum_{i=1}^{N} X_{i}^{\top} M_{\iota} X_{i}, B=\frac{1}{N} \sum_{i=1}^{N} X_{i}^{\top} M_{\iota} X_{i}\left[\beta_{i}-E\left(\beta_{i}\right)\right]$, and $C=\frac{1}{N} \sum_{i=1}^{N} X_{i}^{\top} M_{\iota} u_{i}$.

In the traditional panel data model, the parameters are assumed to be homogeneous (meaning $B=0$ ), so that the asymptotic distribution of the fixed effects estimator is determined by understanding the terms $A$ and $C$. From the representation in equation (3), the term that potentially renders the fixed effects estimator inconsistent is given by

$$
B=\frac{1}{N} \sum_{i=1}^{N} X_{i}^{\top} M_{\iota} X_{i}\left[\beta_{i}-\mathrm{E}\left(\beta_{i}\right)\right]
$$

Intuitively, if the slope parameters $\beta_{i}$ are correlated with the variation in $X_{i}$ in the crosssection, fixed effects estimation will not be consistent. ${ }^{2}$ In fact, one can see that fixed effects is consistent if and only if $B \stackrel{p}{\rightarrow} 0$ under the conditions that allow $C \stackrel{p}{\rightarrow} 0$.

\footnotetext{
${ }^{2}$ Pesaran and Smith (1995) show that in a dynamic panel data model, heterogeneity causes bias in any case.
} 
There are conditions where fixed effects may be appropriate. Indeed, the literature proposes conditions where OLS-FE estimators are robust to heterogeneity in the slope parameters (references include Wooldridge $(2003,2005,2010)$ ). In the context of our model, the conditions amount to

$$
\mathrm{E}\left(\beta_{i} \mid M_{\iota} X_{i}\right)=\mathrm{E}\left(\beta_{i}\right)
$$

which would imply that

$$
\mathrm{E}\left(X_{i}^{\top} M_{\iota} X_{i}\left[\beta_{i}-\mathrm{E}\left(\beta_{i}\right)\right]\right)=0,
$$

and then (4) would converge to zero under suitable regularity conditions. In other words, the assumption such as (5) implies fixed effects estimation is consistent.

Nevertheless, in general, the OLS-FE can be inconsistent. Consider the representation of the bias term in (3). A simple application of the law of large numbers suggests that the asymptotic bias is

$$
\mathrm{E}\left(X_{i}^{\top} M_{\iota} X_{i}\right)^{-1} \mathrm{E}\left(X_{i}^{\top} M_{\iota} X_{i}\left[\beta_{i}-\mathrm{E}\left(\beta_{i}\right)\right]\right) .
$$

To illustrate how this might arise in a simple empirical model, consider the standard linear model with two covariates,

$$
y_{i t}=\alpha_{i}+w_{i t} \theta_{i}+z_{i t} \gamma_{i}+u_{i t} .
$$

The representation of the asymptotic bias for (3) considering the simple model with two covariates in (6) is given by following equation

$$
\left(\begin{array}{c}
\widehat{\theta}_{F E}-\mathrm{E}\left(\theta_{i}\right) \\
\widehat{\gamma}_{F E}-\mathrm{E}\left(\gamma_{i}\right)
\end{array}\right) \stackrel{p}{\rightarrow}\left(\begin{array}{cc}
\mathrm{E}\left(\sigma_{w i}^{2}\right) & \mathrm{E}\left(\sigma_{w z i}\right) \\
\mathrm{E}\left(\sigma_{w z i}\right) & \mathrm{E}\left(\sigma_{z i}^{2}\right)
\end{array}\right)^{-1}\left(\begin{array}{c}
\mathrm{E}\left[\sigma_{w i}^{2}\left(\theta_{i}-\theta\right)\right]+\mathrm{E}\left[\sigma_{w z i}\left(\gamma_{i}-\gamma\right)\right] \\
\mathrm{E}\left[\sigma_{w z i}\left(\theta_{i}-\theta\right)\right]+\mathrm{E}\left[\sigma_{z i}^{2}\left(\gamma_{i}-\gamma\right)\right]
\end{array}\right)
$$

where $\sigma_{w i}^{2}$ and $\sigma_{z i}^{2}$ represent the individual-specific variance of $w_{i t}$ and $z_{i t}$, respectively, and $\sigma_{w z i}$ is the individual-specific covariance of $w_{i t}$ and $z_{i t}$. What we see from the representation in (7) is that, in general, the covariance between the variance of the regressors and the pa- 
rameters causes the bias. For example, if cross-sectional units with a high variance in $z_{i t}$ have a larger response measured by $\gamma_{i}$, there will be a positive asymptotic bias in the fixed effects estimator for $\gamma$. Moreover, these effects can disseminate to the other parameter, depending on the covariance structure. Nevertheless, equation (7) also shows that the OLS-FE can be asymptotically unbiased when there is no covariance between the variance of the regressors and the parameters. In this case, the elements in the vector in the far right-hand-side are equal to zero. The simple representation in (7) is formalized in the Supplemental Appendix.

\subsection{The Mean Group Estimator: Estimation and Inference}

Our focus is the estimation of models in which response parameters may vary across individuals observed over time (panel data). If response heterogeneity is part of the structural model, one needs to decide what statistic to report. In principle, one needs an estimator that is useful in summarizing heterogeneity in the coefficients of interest, and that at the same time is easy to compute and interpret. In this context, a measure of centrality of the distribution of individuals' responses is arguably a reasonable quantity to estimate. The population parameter of interest is the mean coefficient over the sample individuals, $\mathrm{E}\left(\beta_{i}\right)=\beta$.

The mean group (MG) estimator reports the mean of the regression slope parameters as a way to summarize the population. ${ }^{3}$ For most applications in economics and finance, a viable MG estimator will consist of the average of OLS coefficients from each individual whose data are used to fit an empirical model. This can be represented by

$$
\widehat{\beta}_{M G}=\frac{1}{N} \sum_{i=1}^{N} \widehat{\beta}_{i}
$$

where $\widehat{\beta}_{i}$ is the OLS for each sample individual.

\footnotetext{
${ }^{3}$ This estimator is fully developed in Pesaran (2006) and belongs to the class of minimum distance estimators (see Newey and McFadden (1994)).
} 
The MG estimator allows for both the intercept and slope coefficients to vary across individuals. This happens because, by applying OLS to each individual equation, one simultaneously estimates intercepts (standard "fixed effects") as well as slope coefficients for each individual. Critically, the MG estimator does not suffer from the heterogeneity bias that we characterize in the previous section. That the MG estimator is unbiased stems from the fact that we fit the empirical model onto each individual separately, correctly accounting for individual heterogeneity. The method combines individual estimates equally, averaging over consistent parameters.

Inference for the MG estimator is also straightforward. The asymptotic variance for $\sqrt{N}\left(\widehat{\beta}_{M G}-\beta\right)$ is estimated via

$$
\widehat{\Omega}_{M G}=\frac{1}{N-1} \sum_{i=1}^{N}\left(\widehat{\beta}_{i}-\widehat{\beta}_{M G}\right)\left(\widehat{\beta}_{i}-\widehat{\beta}_{M G}\right)^{\top}
$$

Inference is based on asymptotic normality as the time observations, $T$, and the number of individuals, $N$, increase.

\section{Testing Slope Heterogeneity}

Now we present practical procedures to identify and quantify problem of slope heterogeneity. We first briefly review a test for the presence of slope heterogeneity building on dispersion tests by Pesaran and Yamagata (2008) and Swamy (1970). Importantly, as noted in Section 2 , it is possible that the individual heterogeneity is such that there is no bias under the OLS-FE. Thus, second, we develop a novel test designed to detect precisely when and how slope heterogeneity will cause bias in parameters estimated via OLS-FE.

Testing for slope homogeneity in large panels has become popular. Pesaran and Yamagata (2008) and Blomquist and Westerlund (2013) consider the panel model in equation 
(1), and develop procedures for testing whether $\beta_{i}=\beta$. In particular, let $\beta_{i}$ be the vector of policy parameters in the model, $k \times 1$. They wish to test the following null hypothesis of slope homogeneity across individuals

$$
H_{0}: \beta_{i}=\beta
$$

for some fixed vector $\beta$ for all $i$, against the alternative $H_{1}: \beta_{i} \neq \beta_{j}$ for some $i, j$. The strategy to test the null in (10) is estimate regression coefficients using the time-series for each individual, then compare the estimates with $\beta$.

The Pesaran-Yamagata-Swamy (PYS) test discussed above provides a way to identify the presence of individual slope heterogeneity in a model. Slope heterogeneity, however, need not cause OLS-FE estimates to be biased as shown in equation (7) (see also Wooldridge $(2005,2010))$. Alternatively, the bias could be small enough so as to make the OLS-FE still desirable. We now introduce a novel test measuring the magnitude of the slope heterogeneity bias in OLS-FE estimations.

We wish to test the hypothesis that the heterogeneity across individuals does not induce a bias in the OLS-FE estimator. Equation (5) shows that the OLS-FE estimates are asymptotically biased when the heterogeneous coefficients are related to the variance and covariance of the regressors. The intuition is that the term $X_{i}^{\top} M_{\iota} X_{i}$ captures the variance-covariance structure. Thus, we construct a test based on this result. In particular, we wish to test the null hypothesis that there is no correlation between the coefficients and covariance of the regressors. Formally, the null hypothesis is defined as

$$
H_{0}: \mathrm{E}\left[X_{i}^{\top} M_{\iota} X_{i}\left(\beta_{i}-\beta\right)\right]=0
$$

where $X_{i}=\left(x_{i 1}, \ldots, x_{i T}\right)$. For notational simplicity let $\delta:=\mathrm{E}\left[X_{i}^{\top} M_{\iota} X_{i}\left(\beta_{i}-\beta\right)\right]=0$. Therefore, the proposed procedure seeks to test the population moment conditions in equation (11) 
required for OLS-FE to consistently estimate the relevant parameters in the model. ${ }^{4}$

The test statistic for the null hypothesis in (11) is based on an estimate of equation (4). Define

$$
\begin{aligned}
\widehat{\delta} & =\frac{1}{N} \sum_{i=1}^{N} X_{i}^{\top} M_{\iota} X_{i}\left(\widehat{\beta}_{i}-\widehat{\beta}_{M G}\right) \\
& =\frac{1}{N} \sum_{i=1}^{N}\left(X_{i}^{\top} M_{\iota} X_{i}-\frac{1}{N} \sum_{j=1}^{N} X_{j}^{\top} M_{\iota} X_{j}\right)\left(\widehat{\beta}_{i}-\widehat{\beta}_{M G}\right) \\
& =\frac{1}{N} \sum_{i=1}^{N} \widehat{\delta}_{i},
\end{aligned}
$$

where $\widehat{\delta}_{i}:=\left(X_{i}^{\top} M_{\iota} X_{i}-\frac{1}{N} \sum_{j=1}^{N} X_{j}^{\top} M_{\iota} X_{j}\right)\left(\widehat{\beta}_{i}-\widehat{\beta}_{M G}\right)$, and the second equality holds by the definition of $\widehat{\beta}_{M G}$.

The term $\widehat{\delta}$ is an estimate of the quantity that causes the OLS-FE to be inconsistent. We want to test if that quantity is significantly different from zero. To this end, we need an estimate of its variance, which is calculated using

$$
\widehat{\Omega}=\frac{1}{N} \sum_{i=1}^{N} \widehat{\delta_{i}} \widehat{\delta}_{i}^{\top}
$$

The statistic of interest, which we refer to as the heterogeneity bias $(H B)$ test, is given by

$$
H B=N \times \widehat{\delta}^{\top} \widehat{\Omega}^{-1} \widehat{\delta}
$$

The null hypothesis of the $H B$ test in (11) is that heterogeneity in slope parameters does not cause OLS-FE to be inconsistent. Rejection of the null implies that one should use the MG estimator instead.

\footnotetext{
${ }^{4}$ The test does not depend on other relationships that might exist between $\beta_{i}$ and $X_{i}$. The moment condition depends on the correlation between $\beta_{i}$ and $X_{i}^{\top} M_{\iota} X_{i}$. We thank an anonymous referee for asking us to check this possibility.
} 
Remark 3.1 It is important to highlight the differences between the HB and PYS tests, especially the null hypotheses of interest. The HB test is designed to test a completely different null hypothesis. In particular, the null associated with the $H B$ test in (11) is the lack of correlation between variances of the data and the heterogeneous parameters. On the other hand, the PYS test takes the null hypothesis in equation (10) of no parameter heterogeneity, and detects heterogeneity. Therefore, it is possible to reject the null of parameter heterogeneity with the PYS test, yet fail to reject the null of no bias in the fixed effects estimator. The tests are very much complementary.

Next, we derive the limiting distribution of the proposed $H B$ test. To this end, we consider the following set of assumptions.

Assumption 1 Let the matrix $X_{i}$ have rows $x_{i t}^{\top}$. Suppose that for all $i$, the matrices $\left(X_{i}^{\top} M_{\iota} X_{i}\right)$ are positive definite and bounded, and tends to a non-stochastic invertible matrix $\mathrm{E}\left(X_{i}^{\top} M_{\iota} X_{i}\right)$.

Assumption 2 Suppose that the model is given by (1) with $\mathrm{E}\left(\beta_{i}\right)=\beta$ and $\mathrm{E}\left(u_{i} \mid X_{j}, \beta_{j}\right)=0$ for all $i, j$.

Assumption $3 X_{i}, u_{i}$ and $\beta_{i}$ are independent across $i$. In addition $\left[\left(X_{i}^{\top} M_{\iota} X_{i}\right)\left(\beta_{i}-\beta\right)\right]$ and vec $\left(X_{i}^{\top} M_{\iota} X_{i}\right)$ have finite $2+\kappa$ moments, $\kappa>0$.

Assumption 4 The terms $\left(X_{i}^{\top} M_{\iota} u_{i}\right),\left(X_{i}^{\top} M_{\iota} X_{i}\right)^{-1} X_{i}^{\top} M_{\iota} u_{i}$, and $\left(\beta_{i}-\beta\right)$ are mean zero with finite $2+\kappa$ moments.

Assumptions 1-4 are similar to those employed for fixed effects estimators for cases with fixed T. Assumption 1 allows us to estimate each individual slope parameter, $\beta_{i}$. This condition could be violated for cases with a limited number of time-series observations, $T$, for example. Notice that the conditions do not explicitly require $T \rightarrow \infty$, which is a common 
requirement in the literature, but they requires enough variability in the time-series dimension to be satisfied. Strict exogeneity is implied from Assumption 2, which is important for application of limiting theorems and simplification of the asymptotic distribution of the statistic. Assumption 3 allows us to invoke a central limit theorem if the null hypothesis is true. The first term in Assumption 3 is similar to the standard condition restricting the errors on the cross-section dimension when $T$ is finite (such as Wooldridge (2010)). Assumption 4 is similar, but these terms are explicitly mean zero. This type of assumption is standard in the literature for fixed effects estimators when $T$ is finite. Note that these conditions allow for serially correlated errors as well as heteroskedasticity.

Now we present the asymptotic distributions of the $H B$ test.

Theorem 1 Suppose that Assumptions 1-4 hold and that $H_{0}: \mathrm{E}\left[X_{i}^{\top} M_{\iota} X_{i}\left(\beta_{i}-\beta\right)\right]=0$, then as $N \rightarrow \infty$ with $T$ finite,

$$
H B=N \widehat{\delta}^{\top} \widehat{\Omega}^{-1} \widehat{\delta}^{\rightarrow} \rightarrow \chi_{k}^{2}
$$

where $k$ is the number of regressors in the model.

Theorem 1 provides the asymptotic distribution of new test. Notably, implementation of the proposed heterogeneity test is straightforward. One simply: (I) computes the test statistic, $H B$, using in equation (12); (II) sets the level of significance; and (III) finds the critical values from standard distribution tables. Since $H B$ is asymptotically a chi-square distribution, critical values are tabulated and widely available. The null hypothesis is rejected if the value of the test is outside the interval defined by the critical value of choice.

The limiting distribution of the $H B$ statistic in Theorem 1 is derived if the null hypothesis is true. That is, if $\mathrm{E}\left[X_{i}^{\top} M_{\iota} X_{i}\left(\beta_{i}-\beta\right)\right]=0$ is true, so that the fixed effects estimator is consistent, then the limiting distribution of the $H B$ statistic follows a chi-square distribution. If the above expectation is not zero, then the heterogeneity of $\beta_{i}$ causes an asymptotic bias. 
To this end, we consider the local power of our $H B$ test by allowing a violation of the expectation in the form of

$$
\mathrm{E}\left[X_{i}^{\top} M_{\iota} X_{i}\left(\beta_{i}-\beta\right)\right]=\frac{\gamma}{\sqrt{N}}
$$

where $\gamma$ is a $k \times 1$ vector.

Theorem 2 Suppose that Assumptions 1-4 hold and that (13) holds, then as $N \rightarrow \infty$ with T finite,

$$
H B=N \widehat{\delta}^{\top} \widehat{\Omega}^{-1} \widehat{\delta} \stackrel{d}{\rightarrow} \chi_{k}^{2}\left(\gamma^{\top}\left[\Psi_{1}+\Psi_{2}\right]^{-1} \gamma\right)
$$

where the non-centrality parameter depends on $\Psi_{1}$ and $\Psi_{2}$, which are defined in the Supplemental Appendix.

This result shows that our test is has power against alternatives where fixed effects has (vanishing) asymptotic bias.

We have posited that the MG estimator remains consistent even in the cases where OLS-FE is not consistent. Our arguments were based on the intuition that MG estimators equally weight the individual slope estimators. Given the conditions required for Theorem 1, we formalize this result in the following theorem.

Theorem 3 Suppose that Assumptions 1-4 hold then as $N \rightarrow \infty$ with $T$ finite,

$$
\sqrt{N}\left(\hat{\beta}_{M G}-\beta\right) \stackrel{d}{\rightarrow} N\left(0, \Psi_{3}\right)
$$

where $\Psi_{3}$ is defined in the Supplemental Appendix.

It important to notice that the result in Theorem 3 holds even if $\mathrm{E}\left[X_{i}^{\top} M_{\iota} X_{i}\left(\beta_{i}-\beta\right)\right] \neq 0$. 


\section{Monte Carlo}

We use Monte Carlo simulations to assess the performance of the methods discussed in the previous sections. Our simulations allow for varying degrees of importance assigned to individual slope heterogeneity. The main objective is to assess the finite sample performance of the $H B$ test. We evaluate it in terms of size and power. We also compare the standard OLS (without fixed effects), OLS-FE and MG estimators in terms of inferential bias and efficiency. Our goal is to illustrate the importance of the individual heterogeneity bias, and its impact on different estimation methods under various data scenarios. In doing so, we restrict our attention to the following cases: (1) no heterogeneity bias; (2) heterogeneity affecting one regressor; (3) heterogeneity affecting both regressors. The first case illustrates the case in which OLS-FE is unbiased and gives the size of the tests. The second case shows that one coefficient can be biased while the other remains unbiased. The last case is more general, as both coefficients can be biased. The last two cases provide the power of the tests.

\subsection{Monte Carlo Designs}

We consider a simple data generating process (DGP). The variable $y_{i t}$ is generated by the model

$$
y_{i t}=\alpha_{i}+\theta_{i} w_{i t}+\gamma_{i} z_{i t}+u_{i t}
$$

where $\alpha_{i}$ is the individual-specific intercept term, while $\theta_{i}$ and $\gamma_{i}$ are individual-specific slope coefficients associated with exogenous variables $w_{i t}$ and $z_{i t}$, respectively. The regression error term, $u_{i t}$, is normally distributed with mean zero and variance one.

As discussed in Section 2, fixed effects estimation of the population averages $\mathrm{E}\left(\theta_{i}\right)$ and $\mathrm{E}\left(\gamma_{i}\right)$ will be biased if there is a non-zero covariance between $\theta_{i}$ and the variance of $w_{i t}$, or if there is non-zero covariance between $\gamma_{i}$ and the variance of $z_{i t}$. We model the dependence in $z_{i t}$ and $\gamma_{i}$, and the heterogeneity in $\gamma_{i}$ as follows: $z_{i t}=\alpha_{i}+\epsilon_{i t}^{z}$ and $w_{i t}=1+\epsilon_{i t}^{w}$, where 
$\epsilon_{i t}^{z} \sim N\left(0, \sigma_{z i}^{2}\right), \epsilon_{i t}^{w} \sim N\left(0, \sigma_{w i}^{2}\right) ; \sigma_{z i}^{2}=1+v_{z i}, \sigma_{w i}^{2}=1+v_{w i} ; v_{w i} \sim v_{z i} \sim \chi_{1}^{2} ; \alpha_{i} \sim N(0,1)$ $u_{i t} \sim N(0,1)$

We divide our experiments into two parts. ${ }^{5}$ In the first part, we set $\theta_{i}=1$ for all individuals in order to isolate the effect of just one of the variables having a heterogeneous effect on $y_{i t}$. The parameter $\gamma_{i}$, representing the individual-specific slope, is generated as

$$
\gamma_{i}=1+2 c-c \sigma_{z i}^{2}+d \epsilon_{i}^{\gamma}
$$

where $\epsilon_{i}^{\gamma}$ is $N(0,1)$ and $c=0, \pm 0.5, \pm 1$. The parameter $d$ is given by $d=\left(2-2 c^{2}\right)^{1 / 2}$ so that the variance of $\gamma_{i}$ equals 2 regardless of the correlation between $\sigma_{z i}^{2}$ and $\gamma_{i}$.

The experiments use the parameter $c$ to modulate the importance of individual slope heterogeneity. When $c=0$, there is heterogeneity in $\gamma_{i}$ coming from $\epsilon_{i}^{\gamma}$. However, since $c=0$, the heterogeneity in the slope coefficient $\gamma_{i}$ is not correlated with the variance in the regressor $z_{i t}$, and the fixed effects estimator provides an unbiased estimate of $\mathrm{E}\left(\gamma_{i}\right)$. As $c$ increases in magnitude, the covariance between $\gamma_{i}$ and $\sigma_{z i}^{2}$ increases, leading to biases in the fixed effects estimation of $\mathrm{E}\left(\gamma_{i}\right)$. For example, when $c=1$, the covariance between $\gamma_{i}$ and $\sigma_{z i}^{2}$ is -2 , resulting in negative bias of the fixed effects estimator. Intuitively, the fixed effects estimator assigns more weight to individuals that have smaller slope coefficients $\gamma_{i}$. When $c=-1$, we have the opposite effect, and the fixed effects estimator will be positively biased. Therefore, the parameter $c$ is very important. Under the null hypothesis of slope homogeneity $c=0$, and we obtain the size of the $H B$ test. Then, $c \neq 0$ we have slope heterogeneity that causes bias in OLS-FE, and we are able to examine the power of the proposed $H B$ test.

In the second part of the experiment, we also allow $\theta_{i}$ to be heterogeneous, and generated by $\theta_{i}=1+2 c-c \sigma_{w i}^{2}+d \epsilon_{i}^{\theta}$, where $\epsilon_{i}^{\theta} \sim N(0,1)$. In this set of simulations, by controlling the constant $c$, we are examining the size and power of the tests. Again, when $c=0$ we recover

\footnotetext{
${ }^{5}$ We conducted other experiments where the errors were serially correlated. The results were similar and are available upon request.
} 
size, when $c \neq 0$ we have power of the tests.

To benchmark our results, we estimate the model using traditional OLS, where all individuals are (incorrectly) assumed to have the same intercept and slopes. Under the data generating process above, the OLS estimate of $\mathrm{E}\left(\gamma_{i}\right)$ will be biased since the individualspecific intercept terms are correlated with $z_{i t}$, and OLS is unable to account for individual effects and cross-sectional slope heterogeneity. We also report results for the OLS-FE model that allows for individual intercepts, but as is standard, assumes that the slopes are homogeneous. Finally, we include the mean group estimators. The MG is presented for all comparisons, it allows for individual-specific intercepts as well as slope coefficients.

\subsection{Monte Carlo Results}

We examine the finite sample properties of the methods considering different DGP. We use $N=500$ for the number of individuals and set the number of time observations, $T$, alternatively, to $5,10,20$, and 30 . The number of replications in each experiment is $5,000 .{ }^{6}$ We first report the results for evaluation of the estimators. Then, we describe the results for the tests.

\subsubsection{Results For Bias And RMSE}

\section{Results For Experimental Design 1}

Table 1 reports the bias and RMSE associated with each of the estimators considered in the first experimental design. In the absence of estimation biases, we would expect to find $\mathrm{E}\left(\theta_{i}\right)=1$ and $\mathrm{E}\left(\gamma_{i}\right)=0$. Deviations from these benchmarks measure the degree of bias in the estimated coefficient. Estimators with better inference properties should present low RMSEs.

\footnotetext{
${ }^{6}$ Since the data used in many empirical applications face limitations on the times series dimension, $T$, our main presentation focuses on variations along this dimension. However, in unreported tables we also experiment with variations in the number of individuals, $N$ (e.g., $N=100$ and $N=1,000$ ). These alternative experiments lead to similar inferences and are readily available from the authors.
} 


\section{TABLE 1 ABOUT HERE}

As predicted, the OLS estimator is biased even when $c=0$, and they produce similar results. For example, with $T=5$ the bias for $\mathrm{E}\left(\gamma_{i}\right)$ under OLS is 0.334. As the time dimension, $T$, increases, the OLS bias does not decline. In sharp contrast, the bias under OLS-FE and MG is virtually zero; less than -0.001 in both cases. While the OLS-FE and MG estimators have similar, negligible biases, the MG has the smallest RMSE. The reason that OLS-FE does not have the smallest RMSE for the case of $c=0$ is that it is not efficient even though it is unbiased. The intuition is that if OLS is appropriate in a model with heterogeneity only in intercepts, the random effects model is more efficient. Even though OLS-FE is consistent (unbiased) when $c=0$, it is not efficient. Moreover, MG has smaller variance for this case even though its use is not necessary.

Now we allow for $c \neq 0$. When $c=-0.5$, the covariance between $\gamma_{i}$ and $\sigma_{z i}^{2}$ is positive and we should see a positive bias in the OLS-FE estimator. This is what we see in Table 1. The estimated values of $\mathrm{E}\left(\gamma_{i}\right)$ under OLS-FE are now positively biased with values 0.493 , $0.496,0.494$, and 0.497 for $T=5,10,20$, and 30 , respectively. The bias is insensitive by increases in the times series dimension. At the same time, the OLS estimate is still biased and have the largest RMSEs. The MG estimator performs uniformly better than all of the other estimators, both in terms of bias magnitude and RMSE. Indeed, the MG method produces virtually unbiased estimates.

When $c=0.5$, we see a negative bias in $\mathrm{E}\left(\gamma_{i}\right)$ for OLS-FE. In this case, the bias in OLS is smaller than when $c=-0.5$, which is an artifact of conflicting bias directions from the intercept effects, $\alpha_{i}$, and the slope effects, $\gamma_{i}$. A more interesting observation is that the sign of the OLS-FE bias changes when $c=0.5$. This change highlights the inferential instability of the OLS-FE estimator in the presence of individual slope heterogeneity.

Finally, as we increase the magnitude of the correlation between $\gamma_{i}$ and $\sigma_{z i}^{2}$ via $c=-1$, the bias for OLS-FE is approximately -1 , while that of the MG remains virtually equal to 
zero. That is, under this form of individual slope heterogeneity the OLS-FE suffers from a severe attenuation-like bias that assigns no relevance to estimates associated with the affected variable, even though the variable has a strong predictive power in the true economic model. For $c=1$, the sign of the OLS-FE bias changes, but the magnitudes are similar to those found when $c=-1$. That is, estimates associated with the affected variable are grossly overestimated and appear to be twice as important as they are in the true model. Notably, the magnitudes of these biases are insensitive to $T$.

\section{Results For Experimental Design 2}

We now change the data generating process by also letting $\theta_{i}$ vary across individuals. This new experiment allows one to have correlation between both slope parameters of the model and the variance of the data. The results of these experiments appear in Table 2.

\section{TABLE 2 ABOUT HeRE}

When $c=0$, by design, the variable $w_{i t}$ is uncorrelated with the individual-specific intercept, $\alpha_{i}$, so that $\mathrm{E}\left(\theta_{i}\right)$ should be unbiased. The results in Table 2 confirm this prediction and show approximately unbiased estimates for $\mathrm{E}\left(\theta_{i}\right)$ for the OLS, OLS-FE, and MG estimators. There is, however, a significant bias for $\mathrm{E}\left(\gamma_{i}\right)$ under OLS estimation, and this bias is insensitive to the times series dimension. Finally, both OLS-FE and MG are approximately unbiased for $\mathrm{E}\left(\gamma_{i}\right)$, with MG having the smallest RMSE.

As $c$ increases in magnitude, the bias and RMSE are expected to increase for OLS, and OLS-FE estimators. Table 2 confirms these predictions. The bias of the OLS-FE is significant for both slope coefficients, whereas MG is largely immune to biases, even for short panels. In addition, the RMSE of the MG estimator declines as the time dimension of the panels increases. 
Figure 1: Heterogeneity bias

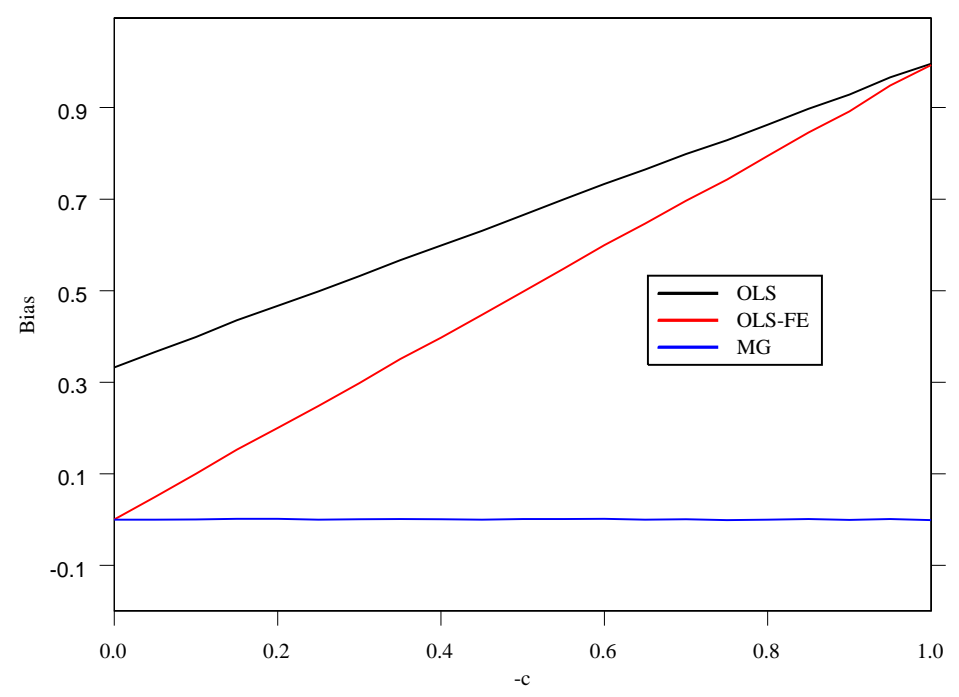

Figure 1 shows the bias of $\mathrm{E}\left(\gamma_{i}\right)$ for OLS, OLS-FE, and MG estimators as a function of the parameter $c$ in the simulations, where $c$ is between 0 and -1 .

\subsubsection{Depicting The Bias}

As a complementary simulation exercise and to illustrate the size of the bias, we reproduce the experiment for $c$ between 0 and -1 , graphing the bias of $\mathrm{E}\left(\gamma_{i}\right)$ as a function of the parameter $c$ for the OLS, OLS-FE, and MG estimators. The results are plotted in Figure 1. One can see that the MG estimator is unaffected by the correlation of the heterogeneous slope parameter, $\gamma_{i}$, with the variance of the data, $\sigma_{z i}^{2}$. The other estimators are, in contrast, very sensitive to heterogeneity in $\gamma_{i}$ as it becomes more correlated with its regressor variance.

\subsubsection{Results For The Heterogeneity Bias Test}

We now explore the use of the $H B$ test in measuring the extent to which slope heterogeneity causes OLS-FE estimates to be biased. We report the empirical size and power of the tests. We use a $5 \%$ nominal size for all computations.

The Experimental designs 1 and 2 assess the finite sample performance of the $H B$ test. 
Figure 2: Power of Heterogeneity Bias test

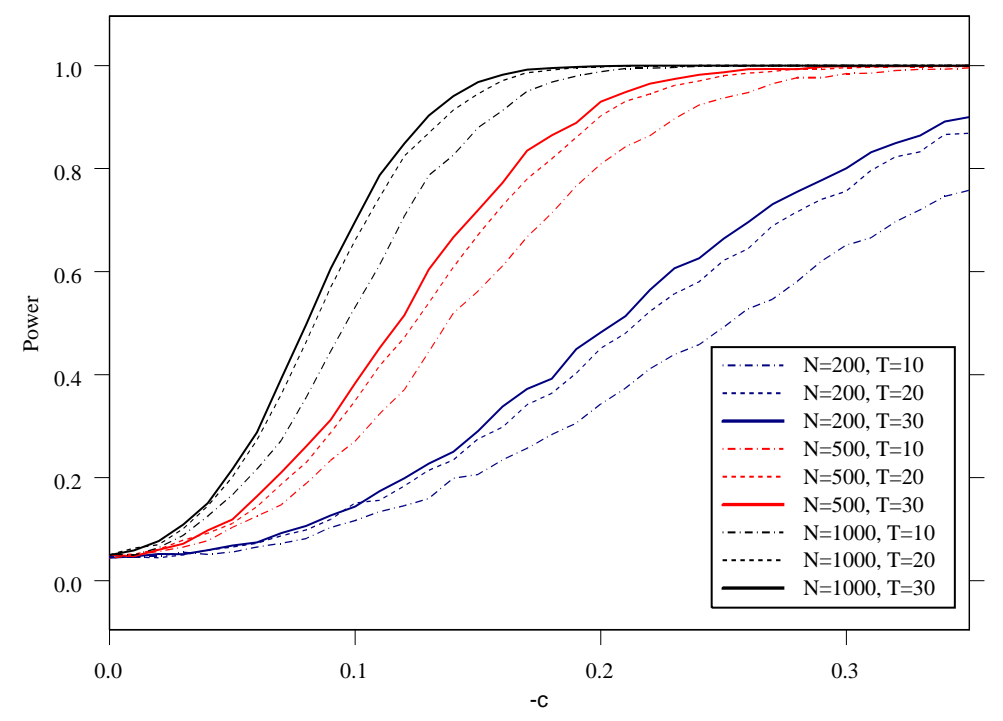

Figure 2 shows the power of the Heterogeneity Bias test as a function of the parameter $c$ in the simulations, where $c$ is between 0 and -1 . For each sample size $N$, power is plotted for $T=10,20$, and 30 respectively.

They produce equivalent results. Thus, we report results only for the first in Figure 2. The results illustrate the costs of parameter heterogeneity. The $H B$ test is applied for $N=200,500$, and 1,000 , as well as values of $T=10,20$, and 30 . When $c=0$ we obtain the size of the test. As the parameter $c$ increases (in absolute values), there is more bias from parameter heterogeneity. For each of the respective sample sizes, we plot the percentage of rejections of the null hypothesis that OLS-FE estimates are unbiased for 5,000 replications.

The results in Figure 2 show that when $c=0$ the empirical size of the $H B$ test approximates the nominal $5 \%$. The results also show strong evidence that the test is very powerful agains deviations for the null hypothesis. The power of $H B$ test improves as the number of individuals, $N$, increases. There is also additional improvement as the number of time periods, $T$, increases. The main point is that as the parameter $c$ increases, the of OLS-FE bias increases, and so does the probability of the $H B$ test rejecting the null of no bias in the OLS-FE estimator. The simulations show the usefulness of our test in detecting cases where 
individual heterogeneity harms inference based on OLS-FE.

To summarize our Monte Carlo experiments, we show that heterogeneity in individual responses to a given economic driver may introduce severe biases in methods commonly used to estimate policy parameters. Focusing on estimators designed to recover individuals' average response (slope) coefficients, we find that the bias of fixed effects estimation can be made arbitrarily large by increasing the magnitude of the covariance between the regression slope and the data variance. The bias can be positive or negative depending on the sign of the covariance. Critically, the mean group estimator we employ is unaffected by the slope heterogeneity bias. In addition, the mean group estimator has the smallest RMSE (hence, the best performance under this inference metric) over the range of our experiments. The bias and RMSE for the MG estimator is uniformly smaller than those of the other estimators. Moreover those statistics are largely insensitive to the time dimension, $T$. Finally, we show that our new $H B$ test has correct empirical size and power to detect precisely the cases where OLS-FE is biased.

\section{Empirical Application: Investment Models}

We illustrate our proposed techniques for estimating models with slope heterogeneity using a traditional corporate finance application. In particular, we compare OLS-FE and MG estimators in the context of the Fazzari et al.'s (1988) investment model. In the model, a firm's investment spending is regressed on a proxy for investment demand (Tobin's $Q$ ) and operating cash flows. A review of the corporate investment literature shows that virtually all empirical work in the area considers panel data models with firm-fixed effects (see, among others, Kaplan and Zingales (1997) and Rauh (2006)). At the same time that there is a consensus about the inclusion of firm-specific intercepts, existing studies assume homogeneous slope coefficients for $Q$ and cash flow across individual firms. ${ }^{7}$

\footnotetext{
${ }^{7} \mathrm{~A}$ number of papers estimate investment-cash flow sensitivities for sample partitions based on proxies for financial constraints (e.g., firm size or existence of bond ratings). These estimations are also subject to
} 
The Fazzari et al. model is commonly represented as

$$
\text { Investment }_{i t}=\alpha_{i}+\theta Q_{i t}+\gamma \text { Cash Flow } \text { Fit }+u_{i t}
$$

where Investment is the ratio of current investment spending scaled by the firm's lagged capital stock, $Q$ is the ratio of the firm's market value over the book value of assets, and Cash Flow is the firm's operating income divided by its lagged capital stock. The parameter $\alpha_{i}$ is the firm-specific fixed effect and $u_{i t}$ is the innovation term.

Suppose that investment is governed by

$$
\text { Investment }_{i t}=\alpha_{i}+\theta_{i} Q_{i t}+\gamma_{i} \text { Cash Flow } i t+u_{i t}
$$

with $\theta_{i}$ and $\gamma_{i}$ possibly different across firms. When data is generated according equation (16), assuming an homogeneous $\theta$ and $\gamma$ across a panel of firms and estimating model (15) may result in severely biased parameters and incorrect inferences. In what follows, we concretely characterize the problems that arise from estimating (15) in lieu of (16).

\subsection{Data Description}

Our data are taken from COMPUSTAT from 1970 through 2010. The sample consists of manufacturing firms with fixed capital of more than $\$ 5$ million (with 1976 as the base year for the CPI). We only study firms whose annual assets and sales growth are less than $100 \%$ (e.g., Almeida and Campello (2007)). To assess the performance of different estimators over the times series dimension, we classify the sample into cases were firms provide, alternatively, a minimum of 10,20 , or 30 years of data.

the firm heterogeneity biases that we highlight in our paper. 


\subsection{Estimation Results}

We estimate the Fazzari et al. model using simple least squares (OLS) and least squares with firm intercepts (OLS-FE). Since papers in the literature capture intertemporal variation by adding time dummies to the OLS method, we also estimate OLS with both firm- and year-fixed effects (OLS-FE2). Moreover, we compare these methods with the random effects (RE), as well as the mean group estimator (MG) examined in Section 2.2.

Before performing our estimations, we test for the presence of slope heterogeneity using the Pesaran-Yamagata-Swamy (PYS) test. We also test for biases in the OLS-FE estimator using the heterogeneity bias $(H B)$ test. The results are displayed in Table 4 . The $p$-values for both tests are less than 0.000 in all specifications we consider. These tests systematically reject the null hypothesis of slope homogeneity and show that slope heterogeneity causes OLS-FE estimates to be inconsistent. That is, rejection by the PYS test implies that there is slope heterogeneity. As discussed earlier in the paper, heterogeneity may or may not cause inconsistency in the OLS-FE estimator. However, since the HB test rejects the null hypothesis, we conclude that OLS-FE is not consistent.

\section{TABle 3 ABOUt HERE}

The results for OLS, RE, OLS-FE, and OLS-FE2 in Table 3 resemble those reported in the literature (e.g., Baker, Stein, and Wurgler (2003), Cummins et al. (2006), and Polk and Sapienza (2009)). The estimates returned across these methods are similar, suggesting that little variation is coming from either firm- or time-specific effects. In a related paper Schaller (1990) examines the empirical performance of investment models, and allows for estimates for the adjustment cost parameters to vary over firms. The results document empirical evidence of heterogeneity across firms. However, Schaller (1990) model does not control for cash flow sensitivity and also does not provide formal tests for slope heterogeneity.

The most salient feature of Table 3 is the difference between the cash flow coefficient 
that is returned by the MG estimator and those returned by the other methods. For the case where we allow firms with 10 or more observations to enter the sample, the cash flow coefficient under the MG method is 0.291 ( $p$-value < 0.001). Under the OLS-FE estimation, that same coefficient is only 0.043. In other words, the MG estimation suggests that the impact of cash flow on investment is about seven times larger than what is implied from the standard OLS-FE. In particular, the OLS-FE estimator implies that $4.3 \%$ of increases in cash flow are directed toward investment, while the MG estimator suggests that $29.1 \%$ of increases in cash flow are invested. Notably, for longer panels, the MG estimates of the cash flow coefficient are about 15 times larger than their OLS-FE counterparts. In all, estimation methods that allow for heterogeneity in firm policies suggest that investment responses to cash flow innovations are about one order of magnitude larger than what is estimated under the OLS-FE framework. The mean group estimation also yields larger coefficients for $Q$, but differences across methods are less notable, implying that cross-firm heterogeneity in investment responses to $Q$ is somewhat less pronounced.

\subsection{Graphical Evidence}

For each sample composition of Table 3, the PYS tests reject the null of slope homogeneity at better than the $0.01 \%$ level. Given the statistical evidence of firm slope heterogeneity, we provide a graphical representation of the distribution of estimated firm coefficients. A histogram of $\gamma_{i}$, the individual firm sensitivity of investment to cash flow, is shown in Figure 3.

Given the evidence that firms are heterogeneous in their responses and the fact that the OLS-FE and MG estimates are very different, one might want to understand and address the nature of the OLS-FE bias. We explore the calculation of the firm heterogeneity bias in the next section, noting that similar calculations can be performed in any other applications considered by the researcher. 
Figure 3: Distribution of Empirical Investment-Cash Flow Sensitivities

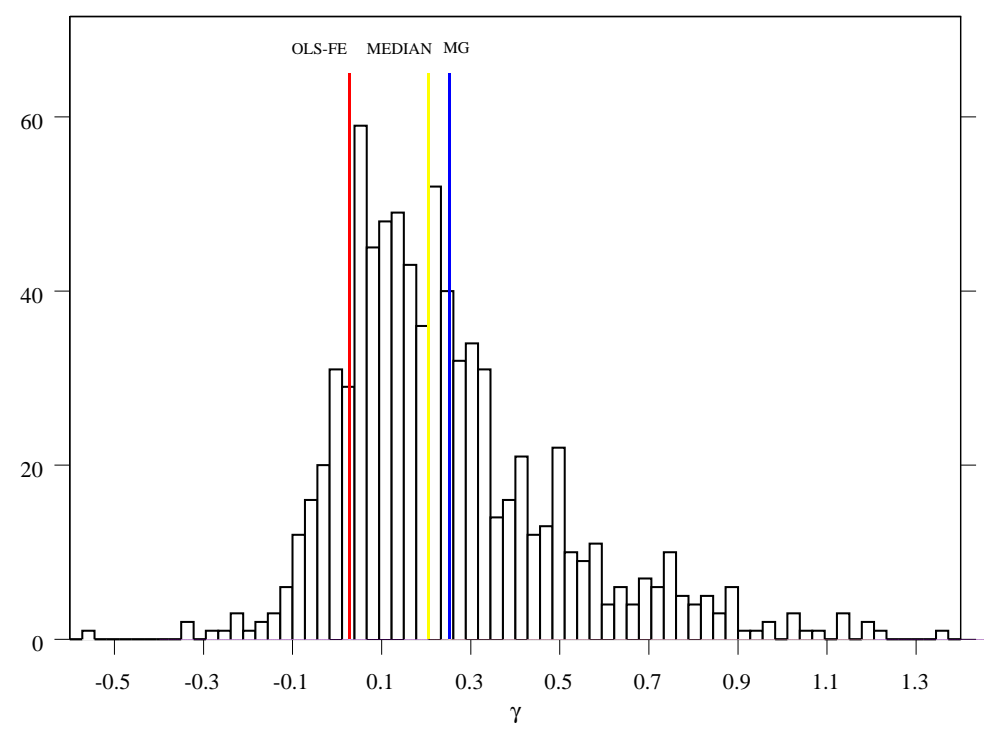

Figure 3 shows the histogram of $\widehat{\gamma}_{i}$, which represent the individual firm sensitivity to cash flow in the investment equation example. The vertical lines are the estimates for OLS-FE, MG and, Median of $\widehat{\gamma}_{i}$.

\subsection{Assessing The OLS-FE Heterogeneity Bias}

The OLS-FE produces negatively biased estimates of cash flow coefficients. Such negative bias would result from a negative correlation between the sensitivity of investment to cash flow, $\gamma_{i}$, and the variance of cash flow, $\sigma_{C F, i}^{2}$. To assess the degree of that correlation, we contrast the distribution of those two parameters in Figure 4. We plot two measures of association. The red line shows a nonparametric smoother capturing local features of the relationship. ${ }^{8}$ Figure 4 shows that there is a strong negative relation between the sensitivity of investment to cash flow and the variance of cash flow for each firm. It is this parameterdata correlation structure that generates the policy heterogeneity bias that arises under the fixed effects framework. Thus, Figure 4 provides an illustration and empirical justification of the conclusion of the $H B$ test, that the slopes are related to the variance of cash flow for the firms, and hence, the $H B$ test rejects the null that fixed effects is appropriate.

\footnotetext{
${ }^{8}$ The smoother we employ is known as the Friedman Super Smoother (see Friedman (1984)).
} 
Figure 4: Investment-Cash Flow Sensitivities and Variance of Cash Flow

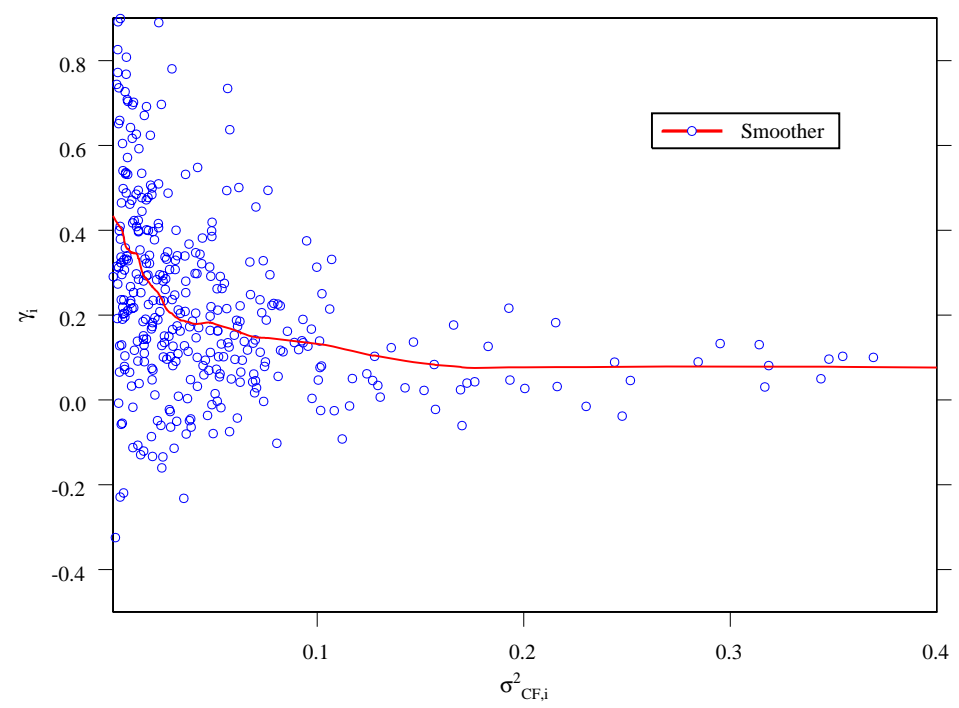

Figure 4 confirms the relationship between $\widehat{\gamma}_{i}$ and $\widehat{\sigma}_{C F, i}^{2}$. The red line represents a smooth nonparametric regression of $\widehat{\gamma}_{i}$ on $\widehat{\sigma}_{C F, i}^{2}$.

\subsection{Investment-Cash Flow Sensitivity Over Time}

Our results have a number of implications for the investment literature. Recent work by Chen and Chen (2012) suggests that the sensitivity of investment to cash flow has declined over time. Critically, the authors argue that point estimates of investment-cash flow sensitivity are approximately zero since the mid 1990's. To show how these inferences are a direct result of the estimation method employed, we present cash flow sensitivity estimates from both OLS-FE and MG estimators. We estimate equation (16) for each year from 1970 to 2010 using a rolling window of 10 years. Rolling estimates of cash flow sensitivities with 95\% confidence bands are presented in Figure 5.

Our results contrast with those of Chen and Chen (2012). Even though we observe a downward trend in investment-cash flow sensitivities, the magnitude of the estimated coefficients are far from zero, and imply economically significant effects through present times. For example, the MG-estimated coefficient for 2006 (which is gauged from the 2001-2010 
Figure 5: Investment Cash Flow Sensitivity Coefficients

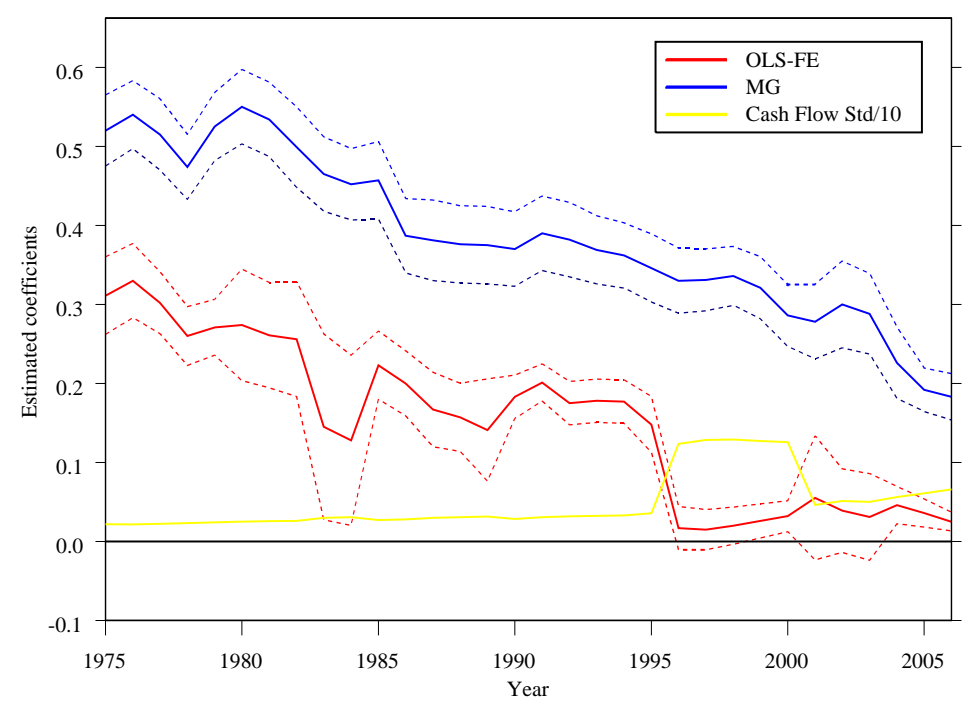

Figure 5 shows the estimated coefficients of $\widehat{\gamma}$ using OLS-FE and MG estimation using a rolling window of $T=10$ time-series observations for each year. The standard deviation of cash flow divided by 10 is shown for the same rolling window periods.

time window) is 0.183 , which contrasts with the OLS-FE estimate of only 0.024 .

Given the evidence of biases in OLS-FE based estimation in environments with heterogeneous firms, the use of the MG estimator appears to be a robust way to estimate the magnitude of the population parameters in the corporate investment model. Our results show that the sensitivity of investment to cash flow remains - in a statistical sense - a potentially important factor in understanding investment behavior.

\section{Conclusion}

We show that heterogeneity in individual responses to economic stimuli can distort estimates that are commonly reported. The theorems and numerical evidence show that standard fixed effects models may often render biased estimates of individual responses in light of slope heterogeneity. As part of the exploration of the potential bias in fixed effects estimation, we 
propose and analyze new statistical tests that are designed to indicate whether fixed effects is the appropriate estimator. One interpretation of the proposed tests is that they are testing the moment conditions required for fixed effects estimator to be consistent for corresponding population parameters of interest. The tests are simple to calculate, governed by a simple distribution, and shown to be effective in an extensive Monte Carlo experiment.

The importance of our test depends on empirically relevant examples. In our empirical model of firm investment, we reject the null hypothesis of no heterogeneity bias. What this finding means is that fixed effects estimation, which is almost universally employed in this empirical literature, is not appropriate. Moreover, our test suggests the use of MG estimation. The estimated coefficients from MG estimation for the sensitivity of investment to cash flow are roughly seven times as large as those estimated via fixed effects. Our findings illustrate the importance of testing for heterogeneity bias and employing the appropriate estimator for population mean of the regression parameters.

Many questions remain to be investigated. A potential useful extension is to extend the test procedures to accommodate correlation between errors from different cross-sectional units, as well as a form of endogeneity. We leave this extension for future research.

\section{References}

Almeida, H., And M. Campello (2007): "Financial constraints, asset tangibility and corporate investment," Review of Financial Studies, 20, 1429-1460.

Baker, M., J. C. Stein, And J. Wurgler (2003): "When does the market matter? Stock prices and the investment of equity-dependent firms," Quarterly Journal of Economics, 118, 969-1005.

Blomquist, J., And J. Westerlund (2013): "Testing slope homogeneity in large panels with serial correlation," Economics Letters, 121, 374-378. 
Breusch, T., And A. Pagan (1979): "A simple test for heteroskedasticity and random coefficient variation," Econometrica, 47, 1287-1294.

Chen, H., And S. Chen (2012): "Investment-cash flow sensitivity cannot be a good measure of financial constraints: Evidence from the time series," Journal of Financial Economics, 103, $390-410$.

Cummins, J. G., K. A. Hassett, and S. D. Oliner (2006): "Investment behavior, observable expectations, and internal funds," American Economic Review, 96, 796-810.

Fazzari, S., R. G. Hubbard, And B. Petersen (1988): "Financing Constraints and Corporate Investment," Brooking Papers on Economic Activity, 1, 141-195.

Friedman, J. H. (1984): "A variable span scatterplot smoother," Laboratory for Computational Statistics, Stanford University Technical Report No. 5.

Hausman, J. (1978): "Specification tests in econometrics," Econometrica, 46, 1251-1271.

Hsiao, C. (2003): Analysis of Panel Data. Cambridge University Press, New York, New York.

Hsiao, C., And M. H. Pesaran (2008): "Random coefficient models," in Advanced Studies in Theoretical and Applied Econometrics, Vol. 46, The Econometrics of Panel Data: Fundamentals and Recent Developments in Theory and Practice, ed. by L. Matyas, and P. Sevestre. SpringerVerlag, Berlin.

Kaplan, S., And L. Zingales (1997): "Do financing constraints explain why investment is correlated with cash flow?," Quarterly Journal of Economics, 112, 169-215.

Newey, W. K., And D. L. MCFAdden (1994): "Large sample estimation and hypothesis testing," in Handbook of Econometrics, Vol. 4, ed. by R. F. Engle, and D. L. McFadden. North Holland, Elsevier, Amsterdam.

Pesaran, H., R. Smith, And K. S. Im (1996): "Dynamic linear models for heterogeneous panels," in The Econometrics of Panel Data: A Handbook of the Theory with Applications, ed. by L. Matyas, and P. Sevestre, pp. 145-195. Kluwer Academic Publishers, Dordrecht. 
Pesaran, M. H. (2006): "Estimation and inference in large heterogeneous panels with a multifactor error structure," Econometrica, 74, 967-1012.

Pesaran, M. H., And R. Smith (1995): "Estimating long-run relationships from dynamic heterogeneous panels," Journal of Econometrics, 68, 79-113.

Pesaran, M. H., And T. Yamagata (2008): "Testing slope homogeneity in large panels," Journal of Econometrics, 142, 50-93.

Phillips, P., And D. Sul (2003): "Dynamic Panel Estimation and Homogeneity Testing under Cross Section Dependence," Econometrics Journal, 6, 217-259.

Polk, C., AND P. SApienza (2009): "The stock market and corporate investment: A test of catering theory," Review of Financial Studies, 22, 187-217.

RAUH, J. (2006): "Investment and financing constraints: Evidence from the funding of corporate pension plans," Journal of Finance, 61, 33-71.

Schaller, H. (1990): "A re-examination of the q theory of investment using U.S. firm data," Journal of Applied Econometrics, 5, 309-325.

Swamy, P. A. V. B. (1970): "Efficient inference in a random coefficient regression model," Econometrica, 38, 311-323.

Wooldridge, J. M. (2003): "Fixed effects estimation of the population-averaged slopes in a panel data random coefficient model," Econometric Theory, 19, 411-413.

(2005): "Fixed-effects and related estimators for correlated random-coefficient and treatment-effect panel data models," Review of Economics and Statistics, 87, 385-390.

(2010): Econometric Analysis of Cross Section and Panel Data, 2nd Edition. The MIT Press, Cambridge, Maryland. 
Table 1.

OLS, OLS-FE, and MG estimators: heterogeneity in $\gamma_{i}$

\begin{tabular}{|c|c|c|c|c|c|c|c|c|c|c|}
\hline & & & \multicolumn{2}{|c|}{$T=5$} & \multicolumn{2}{|c|}{$T=10$} & \multicolumn{2}{|c|}{$T=20$} & \multicolumn{2}{|c|}{$T=30$} \\
\hline & & & $\mathrm{E}\left(\theta_{i}\right)$ & $\mathrm{E}\left(\gamma_{i}\right)$ & $\mathrm{E}\left(\theta_{i}\right)$ & $\mathrm{E}\left(\gamma_{i}\right)$ & $\mathrm{E}\left(\theta_{i}\right)$ & $\mathrm{E}\left(\gamma_{i}\right)$ & $\mathrm{E}\left(\theta_{i}\right)$ & $\mathrm{E}\left(\gamma_{i}\right)$ \\
\hline \multirow[t]{6}{*}{$c=0$} & \multirow[t]{2}{*}{ OLS } & Bias & 0.000 & 0.334 & 0.000 & 0.333 & 0.000 & 0.333 & 0.000 & 0.335 \\
\hline & & RMSE & 0.039 & 0.345 & 0.028 & 0.344 & 0.020 & 0.343 & 0.016 & 0.344 \\
\hline & \multirow[t]{2}{*}{ OLS-FE } & Bias & 0.000 & -0.001 & 0.000 & 0.000 & 0.000 & 0.001 & 0.000 & 0.001 \\
\hline & & RMSE & 0.036 & 0.096 & 0.024 & 0.086 & 0.016 & 0.081 & 0.013 & 0.082 \\
\hline & \multirow[t]{2}{*}{ MG } & Bias & 0.000 & -0.001 & 0.000 & -0.001 & 0.000 & 0.000 & 0.000 & 0.001 \\
\hline & & RMSE & 0.037 & 0.073 & 0.015 & 0.065 & 0.009 & 0.064 & 0.007 & 0.064 \\
\hline \multirow[t]{6}{*}{$c=-0.5$} & \multirow[t]{2}{*}{ OLS } & Bias & -0.001 & 0.664 & 0.001 & 0.663 & 0.000 & 0.662 & 0.000 & 0.667 \\
\hline & & RMSE & 0.042 & 0.673 & 0.030 & 0.671 & 0.021 & 0.670 & 0.017 & 0.675 \\
\hline & \multirow[t]{2}{*}{ OLS-FE } & Bias & -0.001 & 0.494 & 0.000 & 0.495 & 0.000 & 0.494 & 0.000 & 0.499 \\
\hline & & RMSE & 0.040 & 0.513 & 0.027 & 0.511 & 0.019 & 0.509 & 0.015 & 0.513 \\
\hline & \multirow[t]{2}{*}{ MG } & Bias & 0.000 & -0.001 & 0.000 & 0.000 & 0.000 & -0.001 & 0.000 & 0.002 \\
\hline & & RMSE & 0.037 & 0.071 & 0.015 & 0.065 & 0.009 & 0.063 & 0.007 & 0.064 \\
\hline \multirow[t]{6}{*}{$c=0.5$} & \multirow[t]{2}{*}{ OLS } & Bias & 0.001 & 0.004 & 0.000 & 0.000 & -0.001 & 0.004 & 0.000 & 0.002 \\
\hline & & RMSE & 0.045 & 0.128 & 0.032 & 0.116 & 0.022 & 0.112 & 0.018 & 0.112 \\
\hline & \multirow[t]{2}{*}{ OLS-FE } & Bias & 0.001 & -0.494 & 0.000 & -0.496 & 0.000 & -0.494 & 0.000 & -0.496 \\
\hline & & RMSE & 0.040 & 0.515 & 0.027 & 0.512 & 0.018 & 0.509 & 0.015 & 0.510 \\
\hline & \multirow[t]{2}{*}{ MG } & Bias & -0.001 & 0.001 & 0.000 & -0.001 & 0.000 & 0.001 & 0.000 & 0.000 \\
\hline & & RMSE & 0.037 & 0.074 & 0.015 & 0.066 & 0.009 & 0.064 & 0.007 & 0.064 \\
\hline \multirow[t]{6}{*}{$c=-1$} & \multirow[t]{2}{*}{ OLS } & Bias & 0.000 & 0.994 & 0.000 & 0.995 & 0.000 & 1.000 & 0.000 & 0.994 \\
\hline & & RMSE & 0.051 & 1.009 & 0.036 & 1.008 & 0.025 & 1.013 & 0.021 & 1.006 \\
\hline & \multirow[t]{2}{*}{ OLS-FE } & Bias & 0.000 & 0.990 & 0.000 & 0.992 & 0.000 & 0.998 & 0.000 & 0.990 \\
\hline & & RMSE & 0.052 & 1.016 & 0.035 & 1.014 & 0.024 & 1.019 & 0.019 & 1.010 \\
\hline & \multirow[t]{2}{*}{ MG } & Bias & -0.001 & 0.000 & 0.000 & 0.000 & 0.000 & 0.001 & 0.000 & -0.002 \\
\hline & & RMSE & 0.036 & 0.074 & 0.015 & 0.064 & 0.009 & 0.064 & 0.007 & 0.064 \\
\hline \multirow[t]{6}{*}{$c=1$} & \multirow[t]{2}{*}{ OLS } & Bias & 0.001 & -0.335 & 0.000 & -0.331 & 0.000 & -0.335 & 0.000 & -0.332 \\
\hline & & RMSE & 0.057 & 0.386 & 0.040 & 0.375 & 0.028 & 0.376 & 0.023 & 0.372 \\
\hline & \multirow[t]{2}{*}{ OLS-FE } & Bias & 0.000 & -0.999 & -0.001 & -0.994 & 0.000 & -0.998 & 0.000 & -0.994 \\
\hline & & RMSE & 0.053 & 1.026 & 0.035 & 1.016 & 0.024 & 1.019 & 0.019 & 1.013 \\
\hline & \multirow[t]{2}{*}{ MG } & Bias & 0.000 & -0.001 & 0.000 & 0.000 & 0.000 & -0.001 & 0.000 & -0.001 \\
\hline & & RMSE & 0.036 & 0.072 & 0.015 & 0.066 & 0.009 & 0.064 & 0.007 & 0.064 \\
\hline
\end{tabular}

This table shows the bias and the RMSE associated with the estimation of the model in equation (14) using the OLS, OLS-FE, and MG estimators in simulated panel data in the presence of heterogeneity in the parameter $\gamma_{i}$. The parameter $c$ controls the amount and direction of the heterogeneity bias in $\gamma_{i}$. Sample size is $N=500$ and $T=\{5,10,20,30\}$. The number of replications is 5,000 . 
Table 2.

OLS, OLS-FE, and MG estimators: heterogeneity in $\theta_{i}$ and $\gamma_{i}$

\begin{tabular}{|c|c|c|c|c|c|c|c|c|c|c|}
\hline & & & \multicolumn{2}{|c|}{$T=5$} & \multicolumn{2}{|c|}{$T=10$} & \multicolumn{2}{|c|}{$T=20$} & \multicolumn{2}{|c|}{$T=30$} \\
\hline & & & $\mathrm{E}\left(\theta_{i}\right)$ & $\mathrm{E}\left(\gamma_{i}\right)$ & $\mathrm{E}\left(\theta_{i}\right)$ & $\mathrm{E}\left(\gamma_{i}\right)$ & $\mathrm{E}\left(\theta_{i}\right)$ & $\mathrm{E}\left(\gamma_{i}\right)$ & $\mathrm{E}\left(\theta_{i}\right)$ & $\mathrm{E}\left(\gamma_{i}\right)$ \\
\hline \multirow[t]{6}{*}{$c=0$} & OLS & Bias & 0.002 & 0.331 & -0.001 & 0.333 & 0.002 & 0.331 & -0.001 & 0.334 \\
\hline & & RMSE & 0.102 & 0.345 & 0.090 & 0.344 & 0.086 & 0.341 & 0.083 & 0.344 \\
\hline & OLS-FE & Bias & 0.002 & -0.003 & -0.001 & 0.001 & 0.001 & 0.000 & -0.001 & 0.001 \\
\hline & & RMSE & 0.103 & 0.102 & 0.088 & 0.088 & 0.084 & 0.082 & 0.082 & 0.080 \\
\hline & MG & Bias & 0.000 & -0.001 & 0.000 & 0.000 & 0.001 & 0.001 & 0.000 & 0.000 \\
\hline & & RMSE & 0.075 & 0.074 & 0.064 & 0.065 & 0.064 & 0.064 & 0.064 & 0.064 \\
\hline \multirow[t]{6}{*}{$c=-0.5$} & OLS & Bias & 0.497 & 0.664 & 0.497 & 0.664 & 0.497 & 0.666 & 0.498 & 0.665 \\
\hline & & RMSE & 0.519 & 0.675 & 0.514 & 0.673 & 0.513 & 0.674 & 0.513 & 0.672 \\
\hline & OLS-FE & Bias & 0.497 & 0.495 & 0.498 & 0.497 & 0.497 & 0.499 & 0.498 & 0.497 \\
\hline & & RMSE & 0.519 & 0.517 & 0.514 & 0.514 & 0.512 & 0.514 & 0.513 & 0.511 \\
\hline & MG & Bias & 0.000 & -0.001 & -0.001 & 0.000 & 0.001 & 0.001 & 0.000 & 0.001 \\
\hline & & RMSE & 0.072 & 0.073 & 0.064 & 0.064 & 0.064 & 0.064 & 0.064 & 0.063 \\
\hline \multirow[t]{6}{*}{$c=0.5$} & OLS & Bias & -0.497 & 0.003 & -0.495 & 0.001 & -0.497 & 0.002 & $\begin{array}{l}-0.499 \\
\end{array}$ & 0.000 \\
\hline & & RMSE & 0.519 & 0.129 & 0.513 & 0.121 & 0.513 & 0.118 & 0.513 & 0.114 \\
\hline & OLS-FE & Bias & -0.497 & -0.495 & -0.495 & -0.497 & -0.497 & -0.495 & -0.499 & -0.498 \\
\hline & & RMSE & 0.519 & 0.516 & 0.512 & 0.514 & 0.512 & 0.511 & 0.514 & 0.512 \\
\hline & MG & Bias & -0.001 & 0.001 & 0.001 & 0.000 & 0.000 & 0.000 & -0.001 & 0.000 \\
\hline & & RMSE & 0.073 & 0.072 & 0.065 & 0.065 & 0.064 & 0.064 & 0.065 & 0.064 \\
\hline \multirow[t]{6}{*}{$c=-1$} & OLS & Bias & 0.990 & 0.995 & 0.996 & 0.994 & 0.997 & 0.998 & 0.994 & 0.998 \\
\hline & & RMSE & 1.018 & 1.011 & 1.018 & 1.007 & 1.018 & 1.010 & 1.014 & 1.010 \\
\hline & OLS-FE & Bias & 0.990 & 0.991 & 0.995 & 0.988 & 0.997 & 0.994 & 0.994 & 0.995 \\
\hline & & RMSE & 1.018 & 1.020 & 1.018 & 1.011 & 1.017 & 1.015 & 1.014 & 1.014 \\
\hline & MG & Bias & -0.002 & 0.000 & 0.001 & 0.000 & 0.001 & 0.000 & -0.001 & 0.000 \\
\hline & & RMSE & 0.073 & 0.073 & 0.065 & 0.064 & 0.064 & 0.062 & 0.063 & 0.065 \\
\hline \multirow[t]{6}{*}{$c=1$} & OLS & Bias & -0.991 & -0.328 & -0.993 & -0.331 & -0.992 & -0.332 & -0.998 & -0.329 \\
\hline & & RMSE & 1.018 & 0.381 & 1.015 & 0.376 & 1.013 & 0.375 & 1.019 & 0.369 \\
\hline & OLS-FE & Bias & -0.991 & -0.988 & -0.995 & -0.991 & -0.992 & -0.997 & -0.998 & -0.991 \\
\hline & & RMSE & 1.019 & 1.017 & 1.016 & 1.014 & 1.012 & 1.017 & 1.019 & 1.010 \\
\hline & MG & Bias & 0.001 & 0.000 & 0.001 & 0.000 & 0.001 & -0.001 & -0.001 & 0.000 \\
\hline & & RMSE & 0.074 & 0.073 & 0.065 & 0.065 & 0.064 & 0.065 & 0.065 & 0.062 \\
\hline
\end{tabular}

This table shows the bias and the RMSE associated with the estimation of the model in equation (14) using the OLS, OLS-FE, and MG estimators in simulated panel data in the presence of heterogeneity in the parameters $\theta_{i}$ and $\gamma_{i}$. The parameter $c$ controls the amount and direction of the heterogeneity bias in both $\theta_{i}$ and $\gamma_{i}$. Sample size is $N=500$ and $T=\{5,10,20,30\}$. The number of replications is 5,000 . 
Table 3.

Empirical Results for Model of Investment

\begin{tabular}{|c|c|c|c|c|c|c|}
\hline & Variable & OLS & $\mathrm{RE}$ & OLS-FE & OLS-FE2 & MG \\
\hline \multirow[t]{2}{*}{$T \geq 10$} & $Q$ & $\begin{array}{c}0.034^{* * *} \\
(0.005)\end{array}$ & $\begin{array}{c}0.033^{* * *} \\
(0.004)\end{array}$ & $\begin{array}{c}0.031^{* * *} \\
(0.004)\end{array}$ & $\begin{array}{c}0.034^{* * *} \\
(0.005)\end{array}$ & $\begin{array}{c}0.045^{* * *} \\
(0.006)\end{array}$ \\
\hline & Cash Flow & $\begin{array}{c}0.057^{* * *} \\
(0.021)\end{array}$ & $\begin{array}{l}0.046^{* *} \\
(0.019)\end{array}$ & $\begin{array}{c}0.043^{* *} \\
(0.018)\end{array}$ & $\begin{array}{l}0.041^{* *} \\
(0.017)\end{array}$ & $\begin{array}{c}0.291^{* * *} \\
(0.009)\end{array}$ \\
\hline Obs. & 41254 & PYS $p$-value & 0.000 & $H B p$-value & 0.000 & \\
\hline \multirow[t]{2}{*}{$T \geq 20$} & $Q$ & $\begin{array}{c}0.029^{* * *} \\
(0.004)\end{array}$ & $\begin{array}{c}0.028^{* * *} \\
(0.004)\end{array}$ & $\begin{array}{c}0.027^{* * *} \\
(0.004)\end{array}$ & $\begin{array}{c}0.029^{* * *} \\
(0.004)\end{array}$ & $\begin{array}{c}0.045^{* * *} \\
(0.006)\end{array}$ \\
\hline & Cash Flow & $\begin{array}{c}0.038^{*} \\
(0.020)\end{array}$ & $\begin{array}{c}0.031^{*} \\
(0.017)\end{array}$ & $\begin{array}{c}0.038^{*} \\
(0.016)\end{array}$ & $\begin{array}{c}0.028^{*} \\
(0.015)\end{array}$ & $\begin{array}{c}0.251^{* * *} \\
(0.009)\end{array}$ \\
\hline Obs. & 24125 & PYS $p$-value & 0.000 & $H B p$-value & 0.000 & \\
\hline \multirow[t]{2}{*}{$T \geq 30$} & $Q$ & $\begin{array}{c}0.023^{* * *} \\
(0.002)\end{array}$ & $\begin{array}{c}0.022^{* * *} \\
(0.003)\end{array}$ & $\begin{array}{c}0.022^{* * *} \\
(0.003)\end{array}$ & $\begin{array}{c}0.024^{* * *} \\
(0.004)\end{array}$ & $\begin{array}{c}0.030^{* * *} \\
(0.007)\end{array}$ \\
\hline & Cash Flow & $\begin{array}{l}0.020^{*} \\
(0.011)\end{array}$ & $\begin{array}{l}0.015^{*} \\
(0.009)\end{array}$ & $\begin{array}{l}0.015^{*} \\
(0.008)\end{array}$ & $\begin{array}{l}0.015^{*} \\
(0.008)\end{array}$ & $\begin{array}{c}0.228^{* * *} \\
(0.012)\end{array}$ \\
\hline Obs. & 13764 & PYS $p$-value & 0.000 & $H B p$-value & 0.000 & \\
\hline & $\begin{array}{l}\text { Firm Effects } \\
\text { Time Effects }\end{array}$ & $\begin{array}{l}\text { No } \\
\text { No }\end{array}$ & $\begin{array}{l}\text { No } \\
\text { No }\end{array}$ & $\begin{array}{l}\text { Yes } \\
\text { No }\end{array}$ & $\begin{array}{l}\text { Yes } \\
\text { Yes }\end{array}$ & $\begin{array}{l}\text { Yes } \\
\text { No }\end{array}$ \\
\hline
\end{tabular}

This table shows coefficients and standard errors returned from applying OLS, RE, OLS-FE, OLSFE2, and MG methods to estimate equation (16). Standard errors are in parentheses. p-values for Heterogeniety Bias $(H B)$ test is calculated for each subsample. The data are taken from the annual COMPUSTAT industrial files over the 1970 to 2010 period. See text for details. *, **, and *** represent statistical significance at the $10 \%, 5 \%$, and $1 \%$ levels, respectively. 